\title{
High-Q/V photonic crystal cavities and QED analysis in $3 \mathrm{C}-\mathrm{SiC}$
}

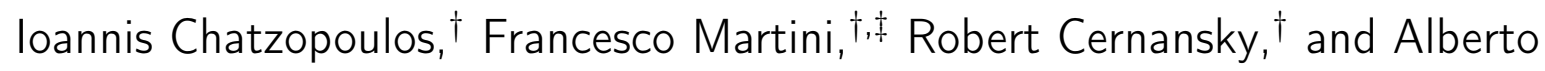 \\ Politi*,
}

School of Physics and Astronomy, University of Southampton, Southampton, SO17 1BJ, United Kingdom

E-mail: A.Politi@soton.ac.uk

\begin{abstract}
Solid state quantum emitters are among the most promising candidates for single photon generation in quantum technologies. However, they suffer from decoherence effects which limit their efficiency and indistinguishability. For instance, the radiation emitted in the zero phonon line (ZPL) of most color centers is on the order of a few percent (e.g. $N V^{-}$centers in Diamond, $V_{S i} V_{C}$ in $S i C$ ) limiting the emission rate of single photons as well as the efficiency. At the same time, reliable interfacing with photons in an integrated manner still remains a challenge in both diamond and $\mathrm{SiC}$ technology. Here we develop photonic crystal cavities with Q factors in the order of 7,100 in 3C SiC. We discuss how this high confinement cavity can significantly enhance the fraction of photons emitted in the ZPL and improve their characteristics. We study the requirements to place $\mathrm{SiC}$ color centers in the strong coupling condition, and analyse the maximum attainable enhancement in the weak coupling regime. The robustness of
\end{abstract}

${ }^{*}$ To whom correspondence should be addressed

${ }^{\dagger}$ School of Physics and Astronomy, University of Southampton, Southampton, SO17 1BJ, United Kingdom

${ }_{\ddagger}^{\ddagger}$ Now at: Istituto di Fotonica e Nanotecnologie (IFN), CNR, via Cineto Romano 42, 00156 Roma, Italy 
the increased efficiency and improved indistinguishability can open the way to quantum technologies in the solid state.

\section{Keywords}

nanophotonics; silicon carbide; light-matter interaction; photonic crystal; Purcell factor; Cavity QED

The field of quantum science and technology offers a unique opportunity for studying the fundamentals of physics while delivering technological innovation. Still, many challenges remain unresolved. The ideal platform for implementing quantum technology experiments would combine a list of features ${ }^{1},{ }^{2}$ including the requirement for addressable and low decoherence qubits, that has been proven hard to satisfy. Among them, the most challenging is the requirement of scalability. Considering this limitation, solid state quantum systems coupled to integrated optical circuits have attracted an increasing amount of interest. To benefit from their capabilities in quantum optics experiments, controllable single photon sources, possibly coupled to a long lived spin state, are required to display high level of indistinguishability while maintaining high efficiencies. Despite recent advancements ${ }^{3},{ }^{4}$ an all inclusive technology remains to be found.

Platforms in solid state have attracted interest, thanks to their more robust spin control and high brightness. These span from quantum dots in GaAs to color centers in 2D materials. ${ }^{5}$ During the last decade, one of the most studied platforms in solid state have been color centers in diamond. In particular, the nitrogen vacancy $\left(\mathrm{NV}^{-}\right)$center and the silicon vacancy $\left(\mathrm{SiV}^{-}\right)$are the most studied. $\mathrm{NV}^{-}$centers in diamond exhibit electronic spin with millisecond coherence times ${ }^{67}$ that can be coherently manipulated with optical and microwave pulses. Despite the advantageous electronic spin coherence, the $\mathrm{NV}^{-}$center emits just a small portion of its radiation in the ZPL (Debye-Waller factor $\sim 5 \%$ ), ${ }^{8}$ limiting significantly the brightness of the source and requiring enhancement of the fraction of pho- 
tons emitted in the ZPL. The $\mathrm{SiV}^{-}$on the contrary, exhibits an impressive Debye-Waller factor of $\sim 70 \%$ but its electronic spin coherence time is limited to 100 's of ns ${ }^{91011}$ and only cooling below $500 \mathrm{mK}$ with the use of dilution refrigerator can yield coherence times on the order of $10 \mathrm{~ms} .{ }^{12}$ While remarkable attempts have been made to interface $\mathrm{NV}^{-}$centers with integrated circuitry ${ }^{1314},{ }^{15}$ the fabrication of diamond photonic components remains challenging, while the considerable cost of the material along with the optical wavelength of the $\mathrm{NV}^{-}$center ZPL makes diamond a demanding material for scalable operation.

Alternative solutions have been pursued on more fabrication friendly materials that still possess some of diamond's advantages. Silicon Carbide has been proven to be among the most promising platforms as an alternative to diamond. ${ }^{16} \mathrm{SiC}$ is a wide bandgap material $(2.36-3.23 \mathrm{eV})$ with relatively high refractive index $(n \approx 2.6)$ while at the same time hosts color centers emitting in the near-infrared. $\mathrm{SiC}$ has an already well established fabrication technology, in particular for CMOS high power electronics. The hexagonal polytypes $(4 \mathrm{H}$, $6 \mathrm{H})$ exhibit high material purity as they are usually grown using homoepitaxial techniques. In nanophotonics though, thin films are required for high confinement. For this purpose sophisticated fabrication techniques have to be used for thin film production, including smart cut $^{17}$ and electrochemical etching. ${ }^{18}$ On the other hand, heteroepitaxial techniques have been developed commercially, offering the access to wafers of cubic (3C) SiC on Si substrates. ${ }^{19}$ The drawback of this technique is an increase of unwanted defects close to the silicon interface that can degrade the material quality of the $\mathrm{SiC}$ film.

Both cubic and hexagonal polytypes host a variety of color centers with properties similar to those of the $N V^{-}$, with the divacancy $\left(V_{S i} V_{C}\right.$ one silicon and one neighboring carbon vacancy in $\mathrm{SiC}$ lattice) being between the most studied. The presence of divacancy centers has been demonstrated in all three polytypes, ${ }^{20}$ showing millisecond coherence times in isolated electron spins ${ }^{2122}$ and coherent control up to room temperature. ${ }^{23}$ The divacancy spin triplet configuration of the ground and excited states $(\mathrm{S}=1)$ and a shelving state that can be addressed for spin initialization makes for a configuration similar to that of the $\mathrm{NV}^{-}$ 
in diamond. The photonic properties are also similar, with a Debye-Waller factor of $\sim 7 \%$ and the optical lifetime of about $\sim 23$ ns. $^{22}$

To use those color centers for quantum technologies, many schemes rely on the generation of pure, indistinguishable photons, requiring that only photons emitted in the ZPL are collected. The small Debye-Waller factor poses a severe limitation that can be overcome by enhancing the ZPL intensity (hence the portion of photons emitted in it) and lifetime (consequently increasing the count rate, leading to brighter sources). Placing an emitter inside a cavity leads to an enhancement of the spontaneous emission (SE) rate of the emitter expressed by the Purcell factor

$$
F_{P}=\frac{\gamma_{e n}}{\gamma_{r a d}}=\frac{3}{4 \pi^{2}}\left(\frac{\lambda_{0}}{n}\right)^{3} \frac{Q}{V} \xi^{2}
$$

with $\gamma_{e n}$ being the enhanced by the cavity SE rate, $\gamma_{\text {rad }}$ the radiative SE rate of the defect in bulk material, $\lambda_{0}$ the resonant wavelength of the cavity mode, $n$ the refractive index of the material and $Q / V$ the ratio of the cavity's quality factor over the cavity's mode volume. The parameter $\xi \in[0,1]$ describes the overlap of the defect's dipole with the cavity mode.

Various attempts of fabricating photonic cavities with high $Q / V$ that would enhance light-matter interaction have been made so far in $\mathrm{SiC}^{24252618}{ }^{27}$ spanning the visible to near-IR. However, limited performances have been demonstrated for cavities operating at the divacancy's ZPL wavelength, showing a maximum $\mathrm{Q}=1500$ in $3 \mathrm{C} \mathrm{SiC}^{28} .{ }^{29}$ Efforts in the telecommunication spectrum $(1550 \mathrm{~nm})$ have given remarkable results ${ }^{30}$ and are of high importance in fully photonic schemes, whereas more efforts are needed on developing devices in the spectral vicinity of color centers available in $\mathrm{SiC}$. In this Letter we report the simulation and fabrication of optimized L3 photonic crystal cavities (PhCCs) in 3C SiC. Simulated results show a $Q / V \sim 500,000(n / \lambda)^{3}$, while the samples fabricated were measured to have Q factors as high as 7,100. 


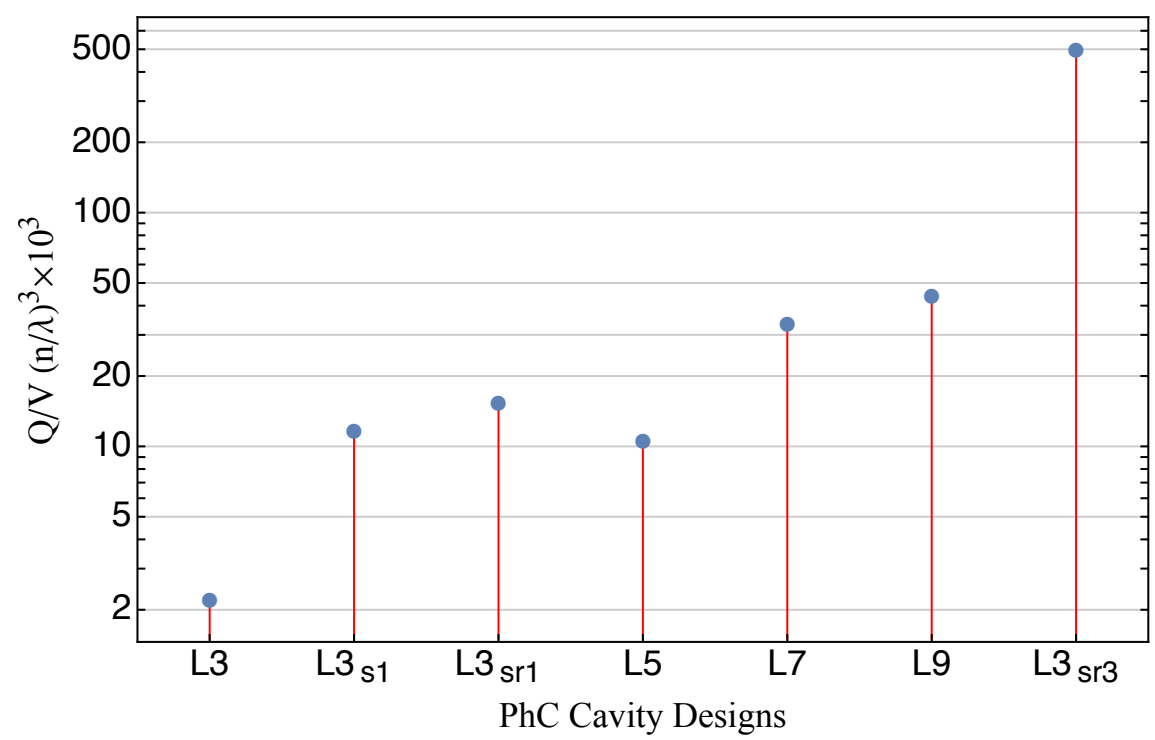

Figure 1: The simulated $Q / V$ ratio for various $\mathrm{PhCC}$ designs in $\mathrm{SiC}(\mathrm{n}=2.6)$. All designs are inducing a linear defect $(\mathrm{L})$ to the photonic crystal. The $x$ in $\mathrm{L} x$ denotes the number of holes omitted, while $s, r, k$ in L $3_{s r k}$ denote that the optimized L3 design includes shifting of holes, reduction of their diameters and the number of holes undergoing modification next to the cavity, respectively.

\section{Simulations}

The Purcell factor $F_{P}$ describes the enhancement of light-matter interaction in a cavityemitter system and is linked to the brightness and quality of the photons produced by the emitter. As can be seen from equation $1, F_{P}$ can be maximized by increasing the $Q / V$ ratio. Longer cavities possess higher $Q$ factors as the portion of the field at the edges, where most of the scattering happens, is decreased. However, longer cavities possess larger modal volume $V$, leading to a small or no gain for the $Q / V$ ratio. Figure 1 shows a comparison of the simulated $Q / V$ ratio for common PhCC designs by 3D finite-difference time-domain (FDTD) in Meep. ${ }^{31}$ Increasing the number of missing holes in the cavity from L3 to L9 produces a sizable increase of the $Q$ factor, so that longer cavities show higher $Q / V$. This trend reaches a plateau as the decrease of the mode at the edge of the cavity does not compensate the increase of modal volume. An alternative approach to maximize $Q / V$ is to optimize the design of the L3 cavity and minimize the scattering losses. As has been described by Noda et. al. work ${ }^{32},{ }^{33}$ the optimization of $Q$ in $\mathrm{PhCC}$ is strongly dependent 


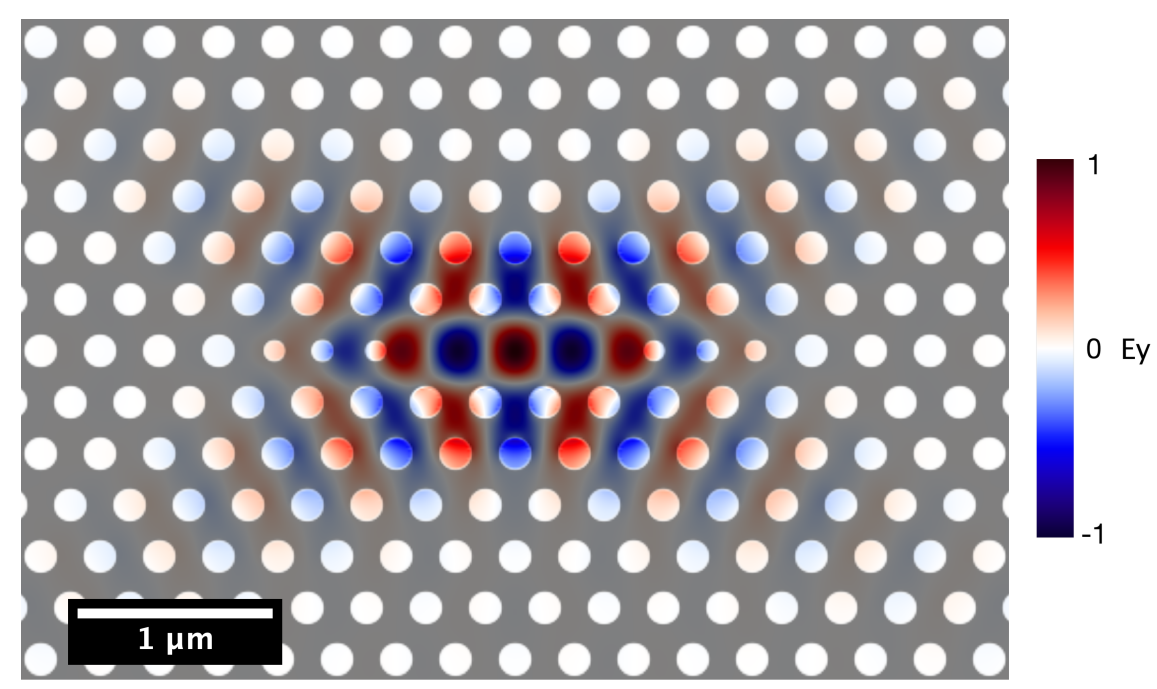

Figure 2: The fundamental mode of the simulated Ey component of the electric field in the optimized L3 $3_{s r 3}$ cavity design in $\mathrm{SiC}$. The parameters of the design are; $r / a=0.2553$, $d / a=0.6, n=2.6, d s_{1} / a=0.3482, d s_{2} / a=0.2476, d s_{3} / a=0.0573, d r_{1} / a=0.098$, $d r_{2} / a=0.0882$ and $d r_{3} / a=0.0927$. The simulated $\mathrm{Q}$ factor of this mode is found to be $\sim 610,000$ while the modal volume $V \sim 1.22(\lambda / n)^{3}$.

on the manipulation of the cavity mode at the edge of the cavity. Scattering at the edge holes, due to the abrupt transition from the cavity to the photonic crystal, leads to an increase of the radiative modes, increasing the losses and hence decreasing considerably the $Q$ factor. For this reason, a smoother transition from the cavity to the photonic crystal regions should be adopted.

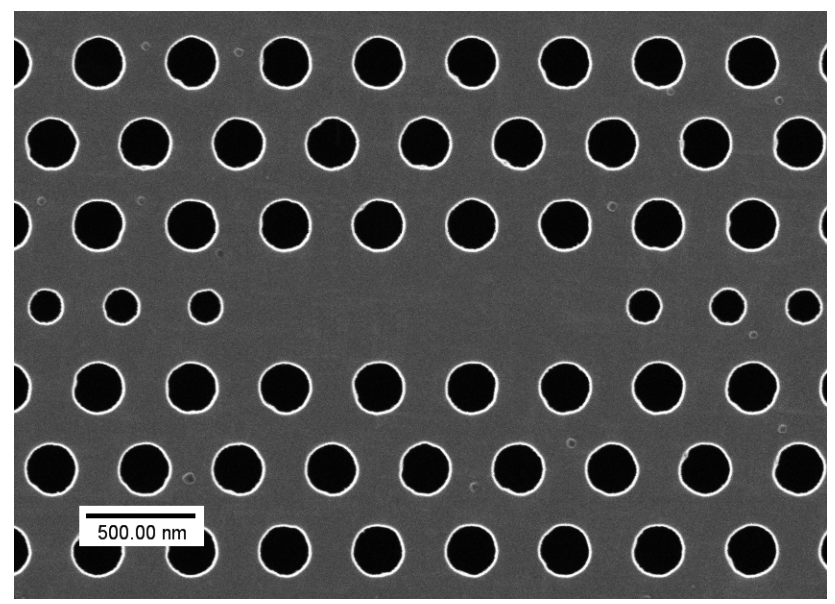

Figure 3: Helium Ion Microscope (HIM) image of the fabricated $\mathrm{L} 3_{s r 3} \mathrm{PhCC}$ design in $3 \mathrm{C}$ $\mathrm{SiC}$. 
Three different optimized L3 designs are examined. The L $3_{s 1}$ design is optimized by shifting the position of one edge hole by $0.21 a$ where $a$ is the lattice constant. Other parameters of the design include $r / a=0.29$ where $r$ the radius of the holes and $d / a=0.6$ where $d$ the slab thickness, while the refractive index used is 2.6. This design shows a simulated $Q$ factor of 9,415 with $V=0.8(\lambda / n)^{3}$, compared to 1,429 and $0.64(\lambda / n)^{3}$ respectively for the bare L3 design. Next L3 ${ }_{s r 1}$ has one hole which has its position shifted and radius reduced on each side of the cavity. The position shift remains $0.21 a$ whilst the radius is reduced by 0.12a. The $Q$ factor of this design is even higher at 14,057 with $\mathrm{V} \sim 0.9(\lambda / n)^{3}$. Finally, the $\mathrm{L} 3_{s r 3}$ is optimized by changing the position and radius of three holes on each side of the cavity. Following similar approach to reference, ${ }^{34}$ a radius $r / a=0.2553$ and thickness of the slab $d / a=0.6$ is used. The three holes are shifted by $0.3482 a, 0.2476 a$ and $0.0573 a$ going closer to further from cavity edge respectively and their radii are reduced by $0.098 a$, $0.0882 a$ and $0.0927 a$. This design gives a quality factor $Q \sim 610,000$ and $V \sim 1.22(\lambda / n)^{3}$. Figure 1 shows the design $Q / V$ for the optimized and bare cavities, highlighting how trading off a smaller modal volume can lead to several orders of magnitude higher $Q / V$ ratio. The simulated fundamental mode (Ey component) of the L $3_{s r 3}$ cavity can be seen in Figure 2; it is possible to notice that the optimized holes are placed where the field intensity is close to zero, minimizing scattering whilst producing high confinement.

\section{Results}

The optimized cavity was fabricated starting from a wafer of 3C SiC heteroepitaxially grown on $\mathrm{Si}\langle 001\rangle$ substrate (NOVASiC) that was thinned down to about $240 \mathrm{~nm}$ by dry etching (a Figure showing the fabrication flow can be found in the Supporting Information (SI).). Next a $100 \mathrm{~nm}$ layer of aluminum and $5 \mathrm{~nm}$ of titanium are deposited as hard mask. The titanium cap layer is used to minimize the formation of aluminum oxide on the mask. CSAR62 resist is spun and exposed with a JEOL JBX-9300FS electron beam lithography system. A 
dry etch step of aluminum transfers the pattern from the resist to the hard mask using a chlorine based ICP-RIE etch. The pattern is then transferred to the $\mathrm{SiC}$ layer thanks to an additional high-power, low pressure ICP-RIE in $\mathrm{SF}_{6}$ gas. The hard mask and two dry etches are optimized to achieve almost vertical walls in the photonic crystal holes. This is particularly important to obtain high- $Q$ cavities, as the hole verticality was suggested to be the most important limit in previously demonstrated L3 cavities operating at $1100 \mathrm{~nm},{ }^{28}$ due to the mixing between TE and TM modes. SEM analysis suggests an etch angle in the holes higher than 86 degrees. In the last fabrication step part of the Si substrate was removed with a TMAH etch, which leaves a $\mathrm{SiC}$ suspended membrane. An example of fabricated L $3_{s r 3}$ design can be seen in Figure 3.

The samples are characterized using a home-build confocal microscope setup, equipped a high NA microscope objective (0.85 Olympus). Resonant scattering measurements are performed with an external cavity tunable laser (New Focus TLB-6723, covering the region $1070-1130 \mathrm{~nm}$ ), which reveals a maximum $Q$ factor of 7,134 (Figure 4) along with simulated modal volume $V \sim 1.22(\lambda / n)^{3}$. Although the $Q$ factor measured here is the highest reported for $3 \mathrm{C} \mathrm{SiC}$, the measured value is significantly smaller than the simulated one, a problem common in photonic crystal demonstrations. Among the critical parameters responsible for this effect, the sensitivity of the design's $Q$ factor to the optimized holes radii and the thickness of the slab have the biggest impact. Another factor to consider is the material losses of $3 \mathrm{C} \mathrm{SiC}^{3536}$ which are estimated to be high in the defect-rich interface with silicon. In particular, it has been shown that the removal of just 100nm of $\mathrm{SiC}$ material close to the silicon interface is sufficient to considerably decrease propagation losses in multimode waveguides. ${ }^{37}$ This effect could be even more significant for photonic crystals, as the SiC layer is thinner to support one single mode in the slab. Moreover, the use of material further away from the Si interface would also increase the optical properties of the divacancies, as well as to decrease the inhomogeneous broadening seen in ensembles. ${ }^{29}$ 


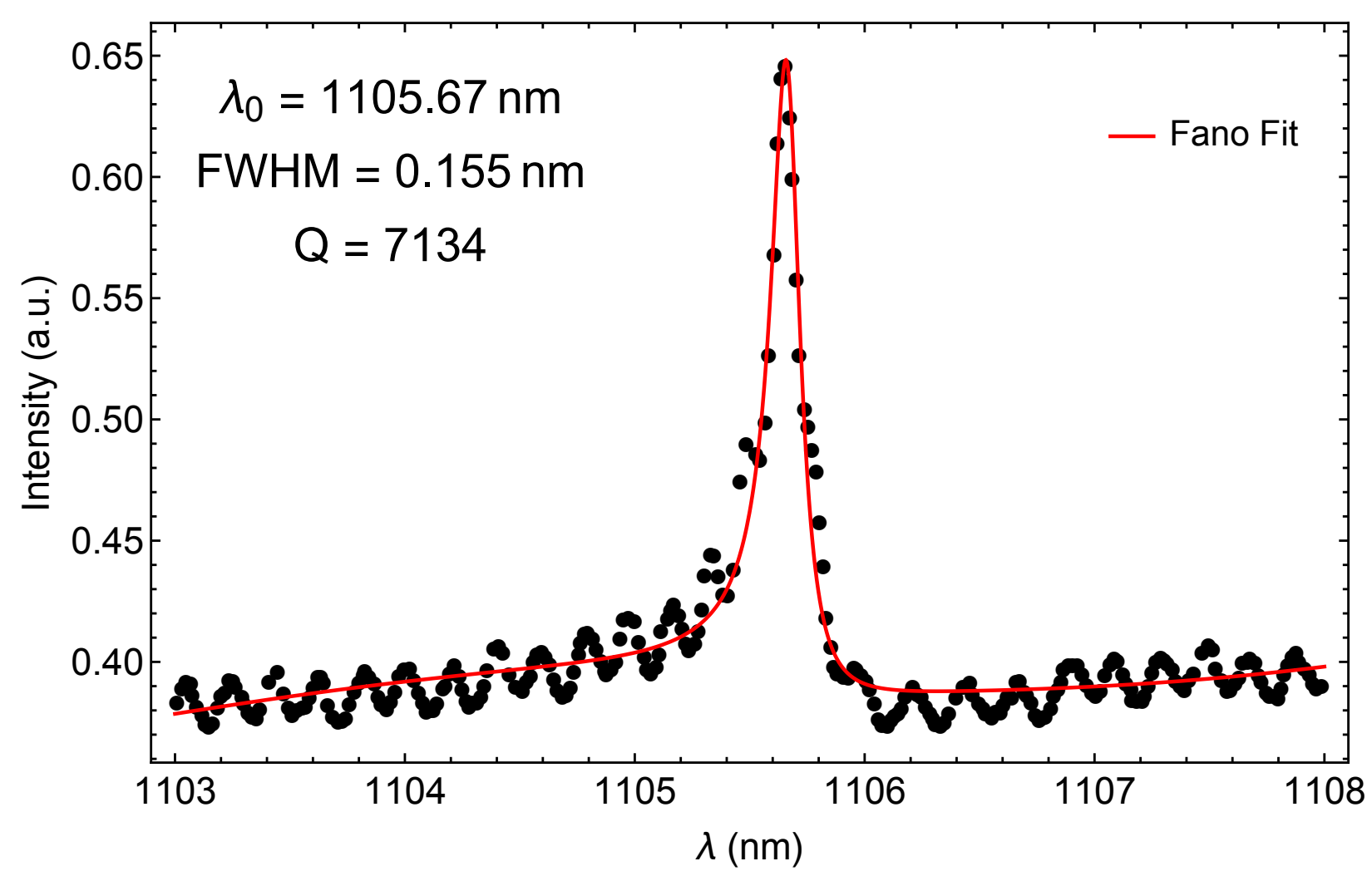

Figure 4: Resonant scattering measurement of a cavity similar to this of Figure 3. A Q factor of 7134 is measured. A Fano type linewidth formula has been used for fitting, a characteristic of resonant scattering technique. The lattice constant used for this cavity was $359 \mathrm{~nm}$. 


\section{Discussion}

From the measured $Q$ factor and the simulated modal volume we can estimate that a Purcell factor of 443 could be achieved with the current sample, in the case of color centers perfectly aligned with the cavity $(\xi=1$ ). (A detailed analysis for non-perfect cavity-emitter alignment can be found in the Supporting Information (SI).) Having integrated circuitry and enhanced on chip light-matter interaction is crucial for experiments in quantum optics and quantum information processing. Besides a higher count rate, the Purcell factor also controls two important factors characterizing systems in these fields: the indistinguishability of the photons generated $(I)$ and the coupling efficiency of the emitter in the cavity mode $(\beta)$. Indistinguishability plays a crucial role in experiments that require two-photon interference and entanglement, while high $\beta$ factor is required for efficient schemes with high emission rates.

As is described by Iles-Smith et.al., ${ }^{38}$ the presence of the phonon sideband decreases significantly both $I$ and $\beta$, limiting the potentials of solid state emitters, such as quantum dots and defects in solids, for scalable quantum optics. This drawback can be overcome by an emitter-cavity system that shows the adequate Purcell enhancement. For a negligible ratio between the cavity linewidth and the phonon sideband width $(0.155 \mathrm{~nm} / \sim 150 \mathrm{~nm}$ in our case), $I$ and $\beta$ can be expressed as,

$$
\begin{gathered}
I=\frac{\Gamma_{t o t}}{\Gamma_{t o t}+2 \gamma_{t o t}}, \\
\beta=\frac{D W \Gamma_{c a v}}{D W \Gamma_{c a v}+\Gamma_{o f f}}
\end{gathered}
$$

with, $\Gamma_{t o t}, \gamma_{t o t}, D W, \Gamma_{c a v}$ and $\Gamma_{o f f}$, being the total emission rate of the emitter, the total dephasing rate, the Debye-Waller factor, the emission rate coupled in the cavity mode and the emission rate not coupled to the cavity mode respectively, while $\Gamma_{t o t}=\Gamma_{\text {cav }}+\Gamma_{\text {off }}$. From 
the definition of $F_{P}(1)$ follows that $\Gamma_{c a v}=\gamma_{e n}=F_{P} \gamma_{r a d}$ and in most cases $\Gamma_{\text {off }}$ can be approximated by $\gamma_{\text {rad }}$. On this basis we can rewrite 2 and 3

$$
\begin{gathered}
I=\frac{F_{P}+1}{F_{P}+1+\frac{2 \gamma_{t o t}}{\gamma_{\text {rad }}}}, \\
\beta=\frac{F_{P}}{F_{P}+1 / D W} .
\end{gathered}
$$

Equations 4 and 5 show the importance of the Purcell factor. Based on the measured $Q \sim 7,134$ and the maximum attainable $F_{P} \sim 443$ the maximum estimated $I$ and $\beta$ values for our system are 0.44 and 0.97 respectively by considering the reported dephasing rate ${ }^{22}$ of $\sim 2 \mathrm{GHz}$ for $3 \mathrm{C} \mathrm{SiC}$. Considerably improved performances can be obtained if the dephasing rate can achieve the values reported for $4 \mathrm{H}$ polytype of $\sim 100 \mathrm{MHz}$, as they would yield $I \sim 0.94$ and $\beta \sim 0.97$. These results do not consider the additional benefit of $2 \mathrm{D} \mathrm{PhCC}$, as the 2D bandgap ( $135 \mathrm{~nm}$ with the current design) can inhibit unwanted emission in the ZPL through the reduction of the optical density of states. ${ }^{39}$ Higher values of $F_{P}$ would further enhance $I$ and $\beta$. For an infinite $F_{P}$ both $I$ and $\beta$ would in principle take near-unity values. In reality, however, a maximum value of $F_{P}$ exists after which the system cannot be described in the perturbative regime. In the strong coupling condition $I$ starts dropping sharply. ${ }^{38}$

The strong coupling regime threshold is usually defined as $g>\kappa / 4, \gamma_{0} / 4$ (see Ref. 40 and a more detailed analysis in the SI), where $g$ denotes the emitter-cavity coupling strength, $\kappa=\omega / Q$ the cavity linewidth and $\gamma_{0}$ the total emitter linewidth. Figure 5 shows the strong coupling condition as $Q$ and $V$ are changed for $\mathrm{SiC}$ color centers compared with experimental results, considering perfect overlap $\xi=1$ and that for color centers $\kappa>>\gamma_{0}$. The figure includes results for both the divacancy as well as for the silicon vacancy in SiC. For this defect, single spin manipulation at room temperature has been also demonstrated ${ }^{41}$ as well 
as magnetometry and thermometry. ${ }^{42}$ It is worth noting that a complete model for the energy transitions of the $\mathrm{SiC}$ colour centres is still missing, so we base the calculations of strong coupling on the radiative and non-radiative rates reported in the literature. The solid black line (dashed blue line) represent the strong coupling threshold for the divacancy (silicon vacancy). With blue are denoted the efforts done in $4 \mathrm{H} \mathrm{SiC}$ at the spectral vicinity of the silicon vacancy, with red efforts done in the vicinity of the divacancy and with open circles the efforts in telecom wavelengths where no defects so far are known in SiC. The divacancy's strong coupling threshold lies higher than the silicon vacancy one, meaning that larger Purcell factors can be obtained. While Ref. 18 lies above the strong coupling line for $4 \mathrm{H}$ silicon vacancies, the cavity studied is not coupled to emitters, and Ref. 27 exploited a lower $Q$ factor cavity $(2,200)$ which lies below the strong coupling threshold.

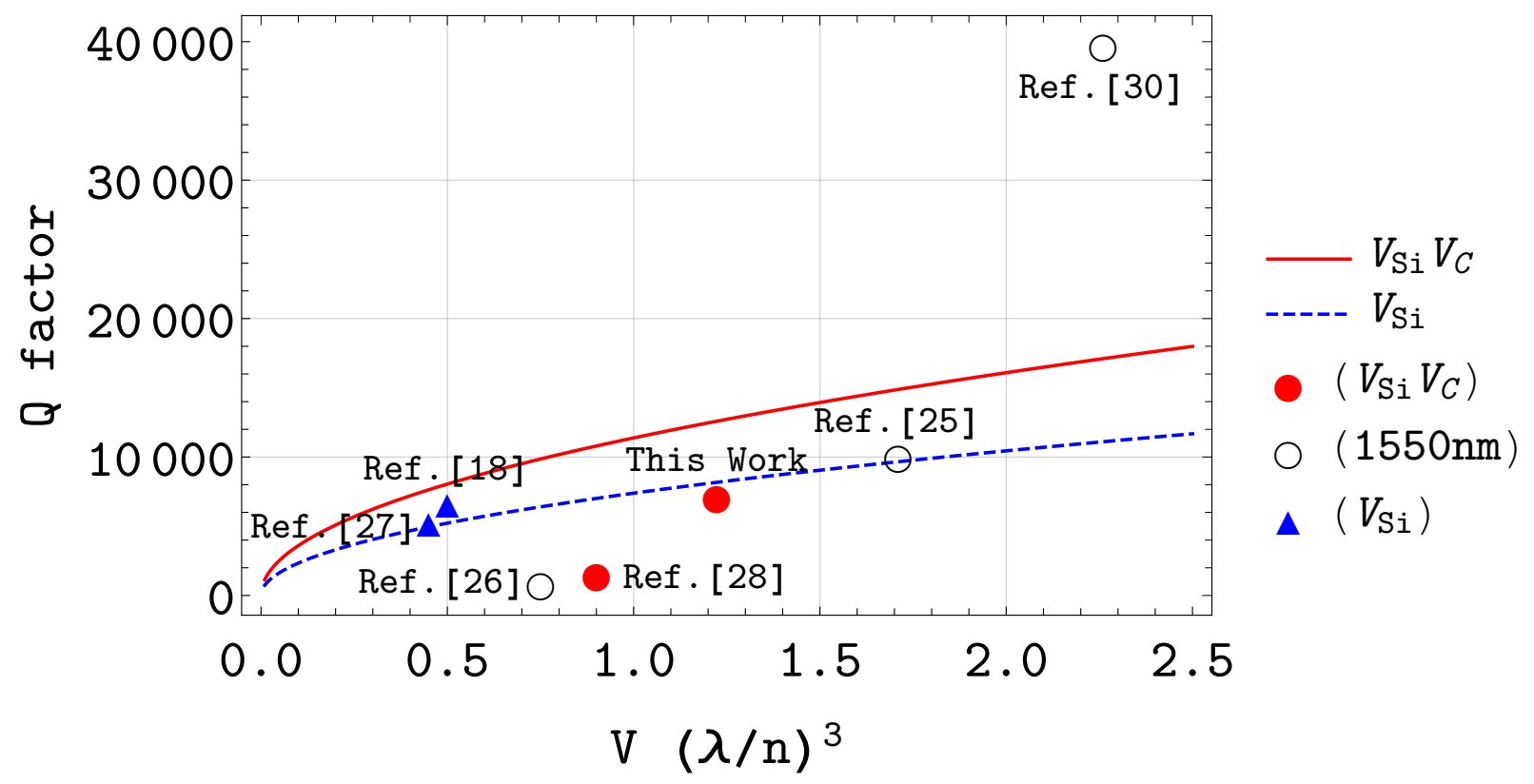

Figure 5: Comparison of experimentally demonstrated $\mathrm{SiC}$ photonic crystal cavities. The solid black (dashed blue) line represents the strong coupling threshold for the divacancy (silicon vacancy) in $\mathrm{SiC}$ assuming perfect emitter-cavity overlap. Red dots denote efforts done in divacancy's spectral vicinity, with both of them being at $3 \mathrm{C} \mathrm{SiC}$. Open circles denote work done in telecom wavelengths with Ref. 25 being in $6 \mathrm{H}$, Ref. 30 in $4 \mathrm{H}$ while Ref. 26 in $3 \mathrm{C} \mathrm{SiC}$. Blue triangles denote efforts done in the vicinity of the silicon vacancy in $4 \mathrm{H}$ $\mathrm{SiC}$.

Further optimization in the fabrication of the $\mathrm{L} 3_{s r 3}$ cavity described here to a $Q \sim 12,600$ 
would put the system at the onset of strong coupling. In this condition the attainable Purcell factor would be 782, corresponding to an increase of $I=0.58$ and $\beta=0.98$ (considering $\gamma_{t o t} \sim 2 \mathrm{GHz}$ for $\left.3 \mathrm{C}\right)$. This highlights that an improvement of the material is required, even with the optimal cavity parameters, if indistinguishable photons are required.
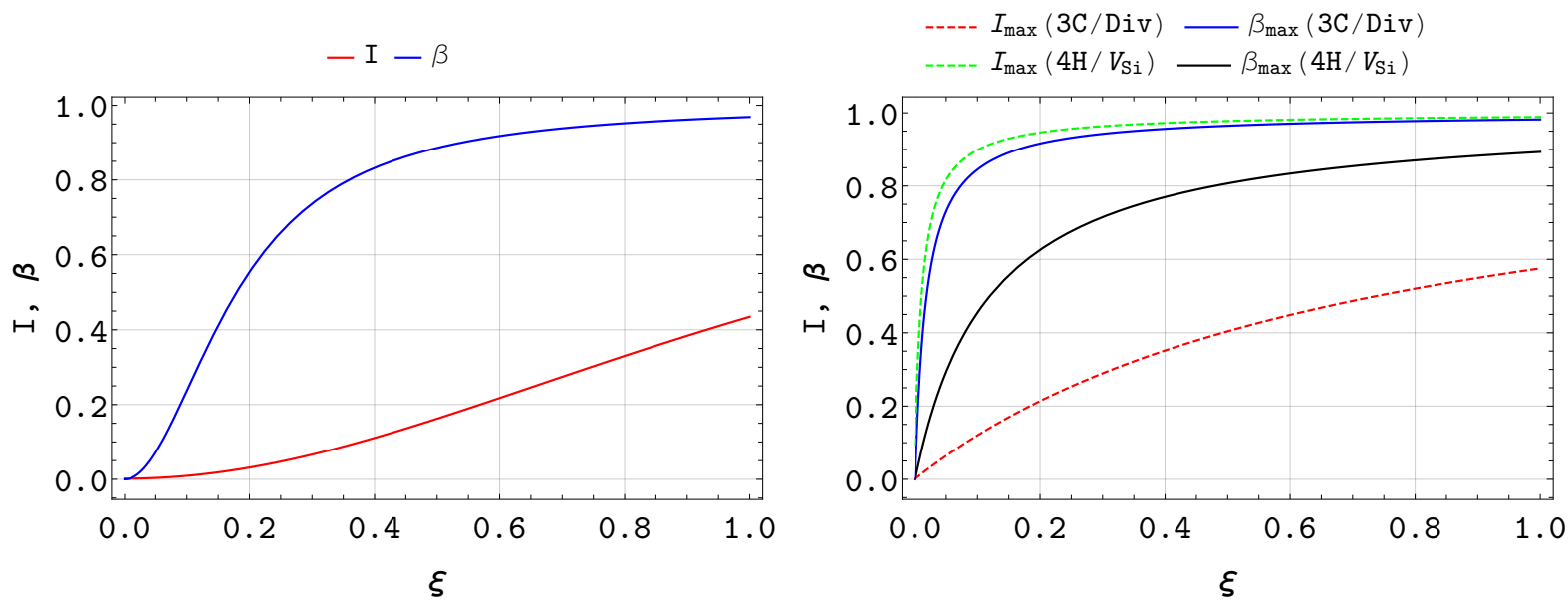

Figure 6: Dependence of emitter-cavity system to the overlap parameter $\xi$. (a) Indistinguishability $I$ and coupling efficiency $\beta$ calculated from the experimentally demonstrated $\mathrm{Q}$ and the dephasing rate of $3 \mathrm{C}-\mathrm{SiC}$ divacancy. (b) $I_{\max }$ and $\beta_{\max }$, corresponding to $Q$ factor at the strong coupling threshold, for the $(3 \mathrm{C} / \mathrm{Div})$ case and $(4 \mathrm{H} / \mathrm{VSi})$ with parameters from Ref. 1843

We finally study how the overlap parameter $\xi$ affects the performances of the emittercavity system. This depends on the spectral, polarization and spatial alignment between emitter and cavity. The spectral overlap can be minimized by tuning the cavity wavelength, either using fabrication techniques, ${ }^{18}$ or reversibly using gas condensation on the photonic structure ${ }^{44}{ }^{27}$ The polarization overlap is estimated as $0.53<\xi<0.93$ depending on the orientation of the particular direction of the divacancy axis to the TE mode of the cavity (see Supporting Information). The spatial overlap can be maximised if targeted ion implantation techniques are used to controllably create the $\operatorname{defect}^{20}{ }^{45}$ in this case $\xi>0.9$ considering a precision of $50 \mathrm{~nm}$. The dependence of $(I, \beta)$ as a function of $\xi$ is reported in Figure 6 . It should be noted that the performances are extremely robust to the changes of $\xi$. This is because the parameters are mainly affected by the dephasing rate of the material and 
the Debye-Waller factor of the emitter. A detailed analysis of the experimental origins and effects of a non-perfect overlap is included in the Supporting Information.

\section{Conclusion}

In this work we have shown, a new PhCC design which yields $Q / V \sim 500,000(n / \lambda)^{3}$ for $\mathrm{SiC}$ devices by shifting the position of three holes on the edge of the cavity and reducing their sizes. Samples fabricated in $3 \mathrm{C} \mathrm{SiC} \mathrm{delivered} \mathrm{a} Q$ factor of 7,134 which can potentially yield high Purcell factors for enhancing light-matter interaction on chip. Improved values of indistinguishability (currently 0.44 for $3 \mathrm{C}$ and 0.99 for $4 \mathrm{H}$ ) and coupling efficiency (0.97) can be achieved for divacancies optimally positioned in the cavity. These values can be further increased with additional improvements in the fabrication and material quality of the $\mathrm{SiC}$ layer. ${ }^{37}$ Indistinguishability is capped at a maximum of $I=0.58$ by the current value of dephasing in $3 \mathrm{C}$ material, as a further increase of $\mathrm{Q}$ factor would put the system in the strong coupling regime. Even if this condition limits the performance of the divacancy as single photon source, it would provide access to non-linear effects at the single photon level $^{44647}$ that can be used for deterministic schemes in quantum computing and simulation. This would make $\mathrm{SiC}$ the first scalable platform to exploit color centers in both the weak and strong coupling conditions. The ability to control the regimes of light-matter coupling will pave the way for future quantum technologies in $\mathrm{SiC}$.

\section{Acknowledgement}

We acknowledge support from the Southampton Nanofabrication Centre. This work was supported by the Engineering and Physical Sciences Research Council (EPSRC) EP/P003710/1 and from the European Union Horizon 2020 research and innovation programme under the Marie Sklodowska-Curie grant agreement No 795923. All data supporting this study are openly available from the University of Southampton repository at 
https://doi.org/10.5258/SOTON/D0996

\section{Supporting Information Available}

Analysis of strong coupling limit for $\mathrm{SiC}$ color centers; effect of the dipole-cavity mode overlap; fabrication details. This material is available free of charge via the Internet at http://pubs.acs.org/.

\section{References}

(1) Ladd, T. D.; Jelezko, F.; Laflamme, R.; Nakamura, Y.; Monroe, C.; O’Brien, J. L. Quantum computers. Nature 2010, 464, 45-53.

(2) DiVincenzo, D. P. The Physical Implementation of Quantum Computation. Fortschr. Phys. 2000, 48, 771-783.

(3) Lodahl, P.; Mahmoodian, S.; Stobbe, S. Interfacing single photons and single quantum dots with photonic nanostructures. Rev. Mod. Phys. 2015, 87, 347-400.

(4) Somaschi, N.; Giesz, V.; de Santis, L.; Loredo, J. C.; Almeida, M. P.; Hornecker, G.; Portalupi, S. L.; Grange, T.; Antón, C.; Demory, J.; Gómez, C.; Sagnes, I.; LanzillottiKimura, N. D.; Lemaítre, A.; Auffeves, A.; White, A. G.; Lanco, L.; Senellart, P. Near-optimal single-photon sources in the solid state. Nat. Photonics 2016, 10, 340345.

(5) Aharonovich, I.; Englund, D.; Toth, M. Solid-state single-photon emitters. Nat. Photonics 2016, 10, 631-641.

(6) Gao, W.; Imamoglu, A.; Bernien, H.; Hanson, R. Coherent manipulation, measurement and entanglement of individual solid-state spins using optical fields. Nat. Photonics 2015, 9, 363-373. 
(7) Balasubramanian, G.; Neumann, P.; Twitchen, D.; Markham, M.; Kolesov, R.; Mizuochi, N.; Isoya, J.; Achard, J.; Beck, J.; Tissler, J.; Jacques, V.; Hemmer, P. R.; Jelezko, F.; Wrachtrup, J. Ultralong spin coherence time in isotopically engineered diamond. Nat. Mater. 2009, 8, 383-387.

(8) Alkauskas, A.; Buckley, B. B.; Awschalom, D. D.; Van de Walle, C. G. First-principles theory of the luminescence lineshape for the triplet transition in diamond NV centres. New J. Phys. 2014, 16, 073026.

(9) Rogers, L. J.; Jahnke, K. D.; Metsch, M. H.; Sipahigil, A.; Binder, J. M.; Teraji, T.; Sumiya, H.; Isoya, J.; Lukin, M. D.; Hemmer, P.; Jelezko, F. All-Optical Initialization, Readout, and Coherent Preparation of Single Silicon-Vacancy Spins in Diamond. Phys. Rev. Lett. 2014, 113, 263602.

(10) Jahnke, K. D.; Sipahigil, A.; Binder, J. M.; Doherty, M. W.; Metsch, M.; Rogers, L. J.; Manson, N. B.; Lukin, M. D.; Jelezko, F. Electron-phonon processes of the siliconvacancy centre in diamond. New J. Phys. 2015, 17, 043011.

(11) Pingault, B.; Jarausch, D.-D.; Hepp, C.; Klintberg, L.; Becker, J. N.; Markham, M.; Becher, C.; Atatüre, M. Coherent control of the silicon-vacancy spin in diamond. Nat. Commun. 2017, 8, 15579.

(12) Sukachev, D. D.; Sipahigil, A.; Nguyen, C. T.; Bhaskar, M. K.; Evans, R. E.; Jelezko, F.; Lukin, M. D. Silicon-vacancy spin qubit in diamond: A quantum memory exceeding 10 ms with single-shot state readout. Phys. Rev. Lett. 2017, 119, 223602.

(13) Wan, N. H.; Mouradian, S.; Englund, D. Two-dimensional photonic crystal slab nanocavities on bulk single-crystal diamond. Appl. Phys. Lett. 2018, 112, 141102.

(14) Burek, M. J.; Chu, Y.; Liddy, M. S.; Patel, P.; Rochman, J.; Meesala, S.; Hong, W.; Quan, Q.; Lukin, M. D.; Lončar, M. High quality-factor optical nanocavities in bulk single-crystal diamond. Nat. Commun. 2014, 5, 5718. 
(15) Faraon, A.; Santori, C.; Huang, Z.; Acosta, V. M.; Beausoleil, R. G. Coupling of nitrogen-vacancy centers to photonic crystal cavities in monocrystalline diamond. Phys. Rev. Lett. 2012, 109, 033604.

(16) Weber, J.; Koehl, W.; Varley, J.; Janotti, A.; Buckley, B.; Van de Walle, C.; Awschalom, D. D. Quantum computing with defects. Proc. Natl. Acad. Sci. 2010, 107, 8513-8518.

(17) Cioccio, L. D.; Letertre, F.; Tiec, Y. L.; Papon, A.; Jaussaud, C.; Bruel, M. Silicon carbide on insulator formation by the Smart-Cut process. Mater. Sci. Eng. 1997, 46, $349-356$.

(18) Bracher, D. O.; Hu, E. L. Fabrication of high-Q nanobeam photonic crystals in epitaxially grown 4H-SiC. Nano Lett. 2015, 15, 6202-6207.

(19) Bosi, M.; Attolini, G.; Negri, M.; Frigeri, C.; Buffagni, E.; Ferrari, C.; Rimoldi, T.; Cristofolini, L.; Aversa, L.; Tatti, R.; Verucchi, R. Optimization of a buffer layer for cubic silicon carbide growth on silicon substrates. J. Cryst. Growth 2013, 383, 84-94.

(20) Falk, A. L.; Buckley, B. B.; Calusine, G.; Koehl, W. F.; Dobrovitski, V. V.; Politi, A.; Zorman, C. A.; Feng, P. X.-L.; Awschalom, D. D. Polytype control of spin qubits in silicon carbide. Nat. Commun. 2013, 4, 1819.

(21) Christle, D. J.; Falk, A. L.; Andrich, P.; Klimov, P. V.; Hassan, J. U.; Son, N. T.; Janzén, E.; Ohshima, T.; Awschalom, D. D. Isolated electron spins in silicon carbide with millisecond coherence times. Nat. Mater. 2015, 14, 160-163.

(22) Christle, D. J.; Klimov, P. V.; de las Casas, C. F.; Szász, K.; Ivády, V.; Jokubavicius, V.; Ul Hassan, J.; Syväjärvi, M.; Koehl, W. F.; Ohshima, T.; Son, N. T.; Janzén, E.; Gali, Á.; Awschalom, D. D. Isolated Spin Qubits in SiC with a High-Fidelity Infrared Spin-to-Photon Interface. Phys. Rev. X 2017, 7, 021046. 
(23) Koehl, W. F.; Buckley, B. B.; Heremans, F. J.; Calusine, G.; Awschalom, D. D. Room temperature coherent control of defect spin qubits in silicon carbide. Nature 2011, 479, $84-87$.

(24) Song, B.-S.; Yamada, S.; Asano, T.; Noda, S. Demonstration of two-dimensional photonic crystals based on silicon carbide. Opt. Express 2011, 19, 11084-11089.

(25) Yamada, S.; Song, B.-S.; Upham, J.; Asano, T.; Tanaka, Y.; Noda, S. Suppression of multiple photon absorption in a $\mathrm{SiC}$ photonic crystal nanocavity operating at $1.55 \mu \mathrm{m}$. Opt. Express 2012, 20, 14789-14796.

(26) Radulaski, M.; Babinec, T. M.; Buckley, S.; Rundquist, A.; Provine, J.; Alassaad, K.; Ferro, G.; Vučković, J. Photonic crystal cavities in cubic (3C) polytype silicon carbide films. Opt. Express 2013, 21, 32623-32629.

(27) Bracher, D. O.; Zhang, X.; Hu, E. L. Selective Purcell enhancement of two closely linked zero-phonon transitions of a silicon carbide color center. Proc. Natl. Acad. Sci. 2017, $114,4060-4065$.

(28) Calusine, G.; Politi, A.; Awschalom, D. D. Silicon carbide photonic crystal cavities with integrated color centers. Appl. Phys. Lett. 2014, 105, 011123.

(29) Calusine, G.; Politi, A.; Awschalom, D. D. Cavity-enhanced measurements of defect spins in silicon carbide. Phys. Rev. Appl. 2016, 6, 014019.

(30) Song, B.-S.; Jeon, S.; Kim, H.; Kang, D. D.; Asano, T.; Noda, S. High-Q-factor nanobeam photonic crystal cavities in bulk silicon carbide. Applied Physics Letters 2018, 113, 231106.

(31) Oskooi, A. F.; Roundy, D.; Ibanescu, M.; Bermel, P.; Joannopoulos, J. D.; Johnson, S. G. MEEP: A flexible free-software package for electromagnetic simulations by the FDTD method. Comput. Phys. Commun. 2010, 181, 687-702. 
(32) Akahane, Y.; Asano, T.; Song, B.-s.; Noda, S. High- Q photonic nanocavity in a twodimensional photonic crystal. Nature 2003, 425, 944.

(33) Akahane, Y.; Asano, T.; Song, B.-S.; Noda, S. Fine-tuned high-Q photonic-crystal nanocavity. Opt. Express 2005, 13, 1202-1214.

(34) Vico Triviño, N.; Minkov, M.; Urbinati, G.; Galli, M.; Carlin, J.-F.; Butté, R.; Savona, V.; Grandjean, N. Gallium nitride L3 photonic crystal cavities with an average quality factor of 16900 in the near infrared. Appl. Phys. Lett. 2014, 105, 231119.

(35) Martini, F.; Politi, A. Linear integrated optics in 3C silicon carbide. Opt. Express 2017, 25, 10735-10742.

(36) Martini, F.; Politi, A. Four wave mixing in 3C SiC ring resonators. Appl. Phys. Lett. 2018, 112, 251110.

(37) Fan, T.; Moradinejad, H.; Wu, X.; Eftekhar, A. A.; Adibi, A. High-Q integrated photonic microresonators on 3C-SiC-on-insulator (SiCOI) platform. Opt. Express 2018, 26, 25814-25826.

(38) Iles-Smith, J.; McCutcheon, D. P.; Nazir, A.; Mørk, J. Phonon scattering inhibits simultaneous near-unity efficiency and indistinguishability in semiconductor single-photon sources. Nat. Photonics 2017, 11, 521âĂŞ526.

(39) Fujita, M.; Takahashi, S.; Tanaka, Y.; Asano, T.; Noda, S. Simultaneous inhibition and redistribution of spontaneous light emission in photonic crystals. Science 2005, 308, $1296-1298$.

(40) Andreani, L. C.; Panzarini, G.; Gérard, J.-M. Strong-coupling regime for quantum boxes in pillar microcavities: Theory. Phys. Rev. B 1999, 60, 13276.

(41) Widmann, M.; Lee, S.-Y.; Rendler, T.; Son, N. T.; Fedder, H.; Paik, S.; Yang, L.P.; Zhao, N.; Yang, S.; Booker, I.; Denisenko, A.; Jamali, M.; Momenzadeh, S. A.; 
Gerhardt, I.; Ohshima, T.; Gali, A.; JanzÃl'n, E.; Wrachtrup, J. Coherent control of single spins in silicon carbide at room temperature. Nat. Mater. 2015, 14, 164-168.

(42) Kraus, H.; Soltamov, V.; Fuchs, F.; Simin, D.; Sperlich, A.; Baranov, P.; Astakhov, G.; Dyakonov, V. Magnetic field and temperature sensing with atomic-scale spin defects in silicon carbide. Sci. Rep. 2014, 4, 5303.

(43) Fuchs, F.; Stender, B.; Trupke, M.; Simin, D.; Pflaum, J.; Dyakonov, V.; Astakhov, G. V. Engineering near-infrared single-photon emitters with optically active spins in ultrapure silicon carbide. Nat. Commun. 2015, 6, 7578.

(44) Mosor, S.; Hendrickson, J.; Richards, B.; Sweet, J.; Khitrova, G.; Gibbs, H.; Yoshie, T.; Scherer, A.; Shchekin, O.; Deppe, D. Scanning a photonic crystal slab nanocavity by condensation of xenon. Applied Physics Letters 2005, 87, 141105.

(45) Wang, J.; Zhang, X.; Zhou, Y.; Li, K.; Wang, Z.; Peddibhotla, P.; Liu, F.; Bauerdick, S.; Rudzinski, A.; Liu, Z.; Gao, W. Scalable Fabrication of Single Silicon Vacancy Defect Arrays in Silicon Carbide Using Focused Ion Beam. ACS Photonics 2017, 4, 1054-1059.

(46) Fink, J.; Göppl, M.; Baur, M.; Bianchetti, R.; Leek, P.; Blais, A.; Wallraff, A. Climbing the Jaynes-Cummings ladder and observing its nonlinearity in a cavity QED system. Nature 2008, 454, 315-318.

(47) Englund, D.; Faraon, A.; Fushman, I.; Stoltz, N.; Petroff, P.; Vučković, J. Controlling cavity reflectivity with a single quantum dot. Nature $\mathbf{2 0 0 7 , 4 5 0 , 8 5 7 - 8 6 1 .}$ 


\section{Graphical TOC Entry}

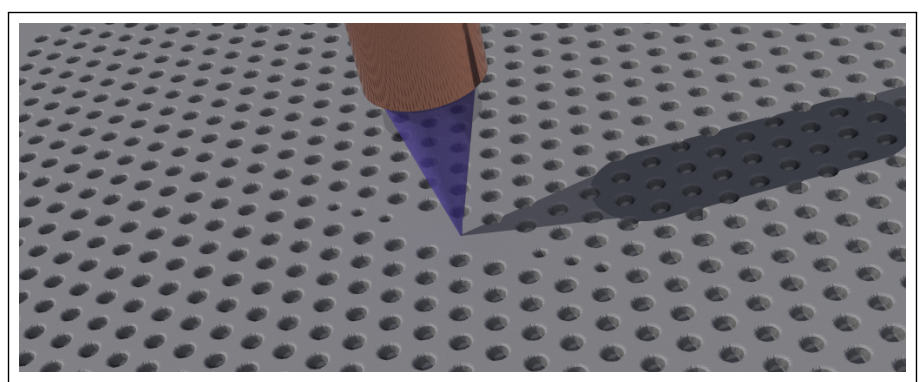

High-Q/V photonic crystal cavities and QED analysis in 3C-SiC

Ioannis Chatzopoulos, Francesco Martini, Robert Cernansky, and Alberto

Politi

Pictorial view of the high-Q/V photonic crystal cavity in silicon carbide 